\title{
Histopathological Study of Gallbladder Lesions in East Singhbhum, Jharkhand
}

\author{
Dr. Silbina Murmu, ${ }^{1}$ Dr.Vijay John Topno ${ }^{2}$, Dr. Budhan Baitha ${ }^{3}$ \\ ${ }^{I}$ (Assistant Professor, Dept. Of Pathology, MGM Medical College Jamshedpur) \\ ${ }^{2}$ (Senior Resident, Dept. Of Surgery, MGM Medical College \& Hospital Jamshedpur) \\ ${ }^{3}$ (Assistant Professor, Dept. Of Pathology, MGM Medical College Jamshedpur)
}

\begin{abstract}
:
Background: The gallbladder is a surgically important organ of the body which may be affected by inflammation, infection, benign and malignant conditions. Gallstones are the commonest biliary pathology and are major cause of morbidity and mortality.

Aims \& Objectives: To study the spectrum of histopathological lesions in gallbladder.

Material \& Method: In this study 106 specimens of gallbladder received in the department of Pathology over a period of two years from September 2013 to September 2015. Clinical details and histological findings were studied and analysed to determine the incidence and prevalence of gallbladder disease.

Result-There were 106 cholecystectomy specimens in which 32 (30.8\%) cases are of males and 74 (69.81\%) cases are of females. Majority of patients was between 41-60 years. Chronic cholecystitis was the commonest condition seen in 79 (74.52) cases. Other benign disease were acute cholecystitis 20 (18.86\%) and cholesterolosi 2 (1.88\%). 2 (1.88\%) cases of gallbladder showed the malignancy.

Conclusion-Commonest lesion of gallbladder is Chronic cholecystitis with gallstones. The cholecystectomy performed for a common conditions like gallstone disease and result in a wide range of histopathological lesions ranging from inflammation, benign conditions to a rare carcinoma of gallbladder.
\end{abstract}

Key words:- cholecystitis, cholecystectomy, gallbladder, gallstone.

\section{Introduction}

Gallbladder is the organ which stores and concentrate bile and helps in digestion of fat(1).The most common pathology found in gallbladder is cholelithiasis followed by cholecystitis. Gallstone afflict $10 \%-20 \%$ of adult population in developed countries (2). The prevalence varies with age, sex and ethnic groups. Most patients were unaware of disease and remain asymptomatic for life. The current changes in lifestyles of individuals pertaining to indulgement in unhealthy fat rich food, lack of exercise, obesity and sedentary lifestyles have once again focused our attention to gallstones and diseases of gallbladder. Now a day a laparoscopic cholecystectomy is the treatment of choice and done routinely for gallstone diseases. It is difficult to diagnose distinctly benign and malignant lesions of gallbladder before surgery without histopathological examination(3). Despite the concept by people that routine histopathology of cholecystectomy specimen plays a dismal role in the management of most patients (4), it plays an important role in clinicopathologic correlation of various lesions of gallbladder and helps in diagnosing premalignant conditions like porcelain gallbladder or malignant conditions like carcinoma in situ and early carcinoma. This is the reason that each and every specimens of cholecystectomy should be evaluated in the histopathology laboratory(5).

On routine histopathologic examination of cholecystectomy specimens, the most commonly found pathology is cholelithiasis. Among other pathology cholecystitis both acute and chronic is common. Cholesterolosis, Xanthogranulomatous cholecystitis, benign polyps, premalignant and malignant conditions are the less common pathological entities.

Cholecystitis associated with cholelithiasis is common disease particularly found in fertile and fatty females in their $4^{\text {th }}$ and $5^{\text {th }}$ decades of age. It can also affect both male and children. This condition has increased in the past two decades due to increase intake of unhealthy fat rich food, lack of exercise, obesity and sedentary lifestyles. Common histopathological findings in chronic cholecystitis are thickening of wall, varying degrees of mononuclear and lymphocytic infiltration and fibrosis(6). The aim of this study is to determine the frequency of various histopathological pattern in cholecystectomy specimens and to emphasize the importance of histopathologic examination of each and every specimen of gallbladder.

\section{Material \& methods}

The present sduty is done in the department of Pathology, M.G.M. Medical college , Jamshedpur over a period of 2 years from September 2013 to September 2015. During the period we received 106 cholecystectomy specimens from Department of Surgery, M.G.M. Medical college, Jamshedpur and 
surrounding hospitals. Cholecystectomy specimens received in the department were fixed in $10 \%$ formalin and submitted to detailed gross examination. Three full thickness sections were obtained from fundus, body and neck of the gallbladder. Additional sections were taken from other grossly abnormal areas if present. Sections were processed and then stained with Haematoxylin and Eosin stain. The stained sections were examined microscopically for a variety of morphological changes in the diseased gallbladder.

\section{Result}

The total number of cholecystectomy specimens studied were 106. Among these 32(30.18\%) were of males and 74(69.81\%) were of females with M:F ratio 1:2.3. In this study, age ranges from 21-70 years with a mean of 46.5 years. Maximum number of patients were between 41-50 years. The age and sex distribution of gallbladder lesions is shown in Table- 1. Gallbladder lesions are predominantly seen in female as compared to the males. All cases were examined microscopically and categorized according to their predominant pattern (Table-2). Out of total 106 cholecystectomy specimens, chronic cholecystitis was the common pathology and was reported in $79(74.52 \%)$ specimens followed by acute on chronic cholecystitis which was reported in 19 (17.92\%) specimens. Other benign lesions were cholesterosis in $2(1.88 \%)$ and Xanthogranulomatous cholecystitis in $01(0.94 \%)$ case. Adenocarcinoma gallbladder was observed in $2(1.88 \%)$ cases.

Table 1:-Age and sex distribution of patients with cholecyctectomy specimens ( $\mathrm{n}=106$ )

\begin{tabular}{|c|c|c|c|}
\hline Age (years) & Males & Females & Total \\
\hline $21-30$ & $1(0.94 \%)$ & $2(1.88 \%)$ & $3(2.83 \%)$ \\
\hline $31-40$ & $8(7.54 \%)$ & $19(17.9 \%)$ & $27(25.47 \%)$ \\
\hline $41-50$ & $15(14.1 \%)$ & $25(23.58 \%)$ & $40(37.73 \%)$ \\
\hline $51-60$ & $6(5.6 \%)$ & $23(21.69 \%)$ & $29(27.35 \%)$ \\
\hline $61-70$ & $2(1.88 \%)$ & $4(3.77 \%)$ & $6(5.66 \%)$ \\
\hline$>70$ & 0 & $1(0.94 \%)$ & $1(0.94 \%)$ \\
\hline Total & $32(30.18 \%)$ & $74(69.81 \%)$ & $106(100 \%)$ \\
\hline
\end{tabular}

Table 2:-Histopathological findings of gallbladder specimens $(\mathrm{n}=106)$

\begin{tabular}{|c|l|c|c|c|c|}
\hline S.No. & $\begin{array}{l}\text { Histopathological } \\
\text { findings }\end{array}$ & $\begin{array}{c}\text { No. of } \\
\text { specimens }\end{array}$ & $\%$ & Males & Females \\
\hline 1 & Chronic cholecystitis & 79 & $74.52 \%$ & 22 & 56 \\
\hline 2 & Acute cholecystitis & 19 & $17.92 \%$ & 8 & 12 \\
\hline 3 & cholesterosis & 2 & $1.88 \%$ & 1 & 1 \\
\hline 4 & $\begin{array}{l}\text { Acute gangrenous } \\
\text { cholecystitis }\end{array}$ & 3 & $2.83 \%$ & 1 & 2 \\
\hline 5 & Adenocarcinoma & 2 & $1.88 \%$ & 0 & 2 \\
\hline 6 & $\begin{array}{l}\text { Xanthogranulomatou } \\
\text { s cholecystitis }\end{array}$ & 1 & $0.94 \%$ & 0 & 1 \\
\hline Total & $\quad----$ & 106 & $100 \%$ & 32 & 74 \\
\hline
\end{tabular}

\section{Discussion}

Gallbladder disease is the most common surgical disorder requiring cholecystectomy either open or laparoscopic technique. Cholecystectomy specimens are received commonly in each histopathology laboratory, where they are evaluated for various pathological lesions. The estimated prevalence of the disease in India is reported to be between $2 \%$ and $29 \%$ (7) . The present study was carried out on 106 cholecystectomy specimens to determine the histopthological spectrum and frequency of gallbladder disease. Histopathology not only establishes a tissue diagnosis in gallstone disease but also contributes towards understanding its etiopathogenesis. It can also help in planning future treatment modality.

In our present study, the age of the patients ranged from 21 to 70 years. Maximum number of patients was in the fourth decade of their life. Male to female ratio is 1:2.3 in the present study. This data showing that females are more affected by the disease of gallbladder. Other studies conducted by Mohan et al(7) and Siddique et al (5) also showed that females to be outnumbered with a male to female ratio of 1:64 and 1:7 respectively.

This high incidence of gallbladder disease in female is due to sedentary life styles and female sex hormones which expose female to the formation of gallstones (7). Chronic cholecystitis was the most common diagnostic entity and $79(74.52 \%)$ cases comes under this group. This entity has various histopathological spectrum like acute on chronic cholecystitis, cholesterosis and porcelain gallbladder. A similar study conducted by Thamil Selvi et al (8) also reported chronic cholecystitis as major histopathological finding to be identified in $67(87 \%)$ cases. In present study cholesterosis was observed in $2(1.88 \%)$ cases. This result is in close approximation to the result of study conducted by Hamidullah Shah et al (9). Xanthogranulomatous cholecystitis was observed in $1(0.94 \%)$ case of cholecystectomy specimens. In this study the incidence was 
slightly lower compared to the study done by Mohan et al(7)who reported an incidence of $2.3 \%$. There is increased wall thickness in Xanthogranulomatous cholecystitis and thus mimics carcinoma on gross examination. Therefore it is important to have awareness of this variant to avoid any aggressive diagnosis like carcinoma(9). In our study, there were $2(1.88 \%)$ cases of carcinoma of the gallbladder. The similar study conducted by Khan et al(10) and Thaml Selvi et al(9) reported the gallbladder carcinoma $2.2 \%$ and $1.2 \%$ respectively. The incidence of gallbladder carcinoma diagnosed during or after a cholecystectomy has been reported to be between $0.19 \%$ and 3.3\%(11). Despite advance in diagnostic and surgical modalities , gallbladder carcinoma is still characterized by late diagnosis and poor prognosis except when incidentally diagnosed at an early stage after cholecystectomy for choleliothiasis.

\section{Conclusion}

The present study revealed that the most common age group for the gallbladder disease is found to be 41-50 years. The females being more affected than males. Chronic cholecystitis was the most common histopathological diagnosis and majority were associated with gallstones. Hardening and thickening of the wall of gallbladder in chronic and Xanthogranulomatous cholecystitis can sometimes mislead towards the diagnosis of malignancy.

\section{References}

[1]. Guyton and Hall textbook of Medical Physiology:588-595.

[2]. Robbins \& Cotran Pathologic Basis of Disease, South Asia Ed.Vol II-875.

[3]. Turkcu G et al. Institutional experience in the histopathological characteristics and frequency of gallbladder lesions. Int. J Clin Exp. Pathol 2016;9:176-80.

[4]. Memon W et al. Histopathological spectrum of gallbladder specimens after cholecystectomy. Pak J Med.Sci. 2011;27:553-6.

[5]. Siddiqui FG et al. Routine histopathology of gallbladder after elective cholecystectomy of gallstones; waste of resources or justified act? BMC Surgery 2013;13:1-4.

[6]. Rosai and Ackerman's Surgical Pathology, ${ }^{\text {th }}$ Ed. Vol I:1041.

[7]. Mohan H et al . Morphological spectrum of gallstone disease in 1100 cholecystectomies in North India. Indian J Surg 2005;67:1402 .

[8]. Thamil Selvi et al. A clinicopathological study of cholecystitis with special reference to analysis of cholelithiasis. International Journal of Basic Medical Science 2011:vol 2, issue 2,68-72.

[9]. Hamidullah Shah et al. Histopathological pattern of 400 cholecystectomy specimens. JPMI VOL30 NO3: $250-3$.

[10]. Khan et al: Spectrum of histopathological lesions in cholecystectomy specimens : Astudy of 360 cases at a teaching hospital in South Delhi. Archives of International Surgery /2013/vol3/issue2: 102-5.

[11]. Zhang WJ, Xu GF, Zou XP, Wang WB et al. Incidental gallbladder carcinoma diagnosed during or after laparoscopic cholecystectomy. World J Surg 2009;33:2651-6. 